\title{
Hierarchical Paired Comparison Modeling, A Cultural Consensus Theory Approach
}

\author{
Pele Schramm, William H. Batchelder \\ University of California, Irvine
}

\begin{abstract}
We introduce a set of models designed to analyze datasets involving responses from multiple subjects on pairwise comparisons from a fixed discrete set of alternatives.These models are part of a greater body of work known as Cultural Consensus Theory (CCT). Like other CCT models, these simultaneously infer each individual's tendency toward aligning with the group consensus, level of agreement on each item, and also a latent consensus value of each alternative. Two primary models are discussed, referred to as the Strong and Weak Consensus Paired-Comparison Models (SCPCM and WCPCM respectively). The SCPCM works under the assumption that all individuals are answering in accordance to the latent consensus values but with varying degrees of accuracy, while the WCPCM relaxes this assumption and assumes minor deviations from latent consensus values in people's average valuation of alternatives. The WCPCM also includes inferences on participants' individual tendencies toward self-consistency (related to their tendencies toward committing violations of transitivity) as well as inferences on the tendency of each item to be evaluated consistently by individuals. The Case III Thurstonian model is used as the
\end{abstract}


backbone for both CPCMs, and inference is conducted under a hierarchical Bayesian framework. Model checks along with applications to both simulated and real data are overviewed.

\section{Introduction}

Paired comparisons are ubiquitous within Psychological science as a means to gain information about people's relative judgments regarding a set of stimuli. Because of the wide usage of paired comparison studies, a principled analysis of such data in a manner that incorporates information gained about differences between both people's response patterns and individual item effects can be useful. Developing an appropriate model to analyze such data is the focus of this paper.

In other item response domains, Cultural Consensus Theory (CCT) has proved useful. CCT is a set of models developed by Romney et al. (1986) that analyze response data by means of assuming one or more sets of latent "ground truths" common among a group of people. In CCT, what is referred to as "ground truth" need not be a reflection of reality, rather it is a reflection of group opinion, although CCT has proven useful also for wisdom of the crowds purposes in some instances as well (e.g. Alexander (2017)). CCT accounts for individual differences in propensity toward answering according to the ground truth, and also accounts for differences in difficulty of evaluating certain items according to the consensus ground truth. CCT uses this information to assess a consensus ground truth while simultaneously providing measures for item difficulty and subject agreement. CCT item response models have been developed for binary response data (Batchelder and Anders, 2012), multiple choice (Romney et al., 1986), continuous closed interval responses (Batchelder 
et al., 2010), and ordinal data (Anders and Batchelder, 2015). For an overview of CCT, see Batchelder et al. (2018).

Thus far, there has not been a CCT model designed for paired comparisons. This paper introduces such a model. Given a complete set of paired comparisons between a group of items (e.g. comparing preference), the goal is to measure consensus attitudes from people's choices. In the spirit of CCT, our model also identifies which people's responses tend to be the closest to consensus, and which items are particularly difficult to assess, and take these things into account in the measurement.

The backbone cognitive model behind our Consensus Paired-Comparison Model (CPCM) is the Thurstonian Model (Thurstone, 1927). The assumptions of the Thurstonian Model are similar in nature to signal detection theory in that the subjective experience of stimuli is assumed to follow a Gaussian distribution on a latent scale. In application to subjective value judgments, for example, a stimuli could be thought to have its esteemed latent value judgment centered at a particular value, and distributed normally. For paired comparisons, the assumption is that each option is evaluated according to its own Gaussian distribution on each trial and the higher-valued option is the one that is selected. Under the assumption of equalvariance Gaussian distributions (referred to as the case V model), the Thurstonian model reduces according to an ordinary probit model. Allowing for separate variances from independent Gaussians gives the Case III model, which is what is used in CPCM, where the probability of choosing option A over option B is

$$
P_{A \succ B}=\Phi\left(\frac{V_{A}-V_{B}}{\sqrt{\sigma_{A}^{2}+\sigma_{B}^{2}}}\right)
$$


Here $\Phi$ denotes the cumulative distribution function of the standard normal distribution, $V_{A}$ and $V_{B}$ denote the mean of the Gaussian distributions of value assessment, whereas $\sigma_{A}^{2}$ and $\sigma_{B}^{2}$ denote their respective variances.

Researchers who utilize the models that we introduce will likely be most interested in finding consensus values for the Vs in equation 1 for each of the options in the set of items. Since we utilize a Bayesian approach, we end up with posterior distributions for these values. Below is a set of inferred posterior distributions of these values measured using the WCPCM (described later) from a data-set in which participants were asked to compare different professions according to which mean salary they believe to be higher. Note that the scale used is not in monetary amounts, rather it is according to the Thurstonian scale which should have a monotonic relationship with salary estimates.

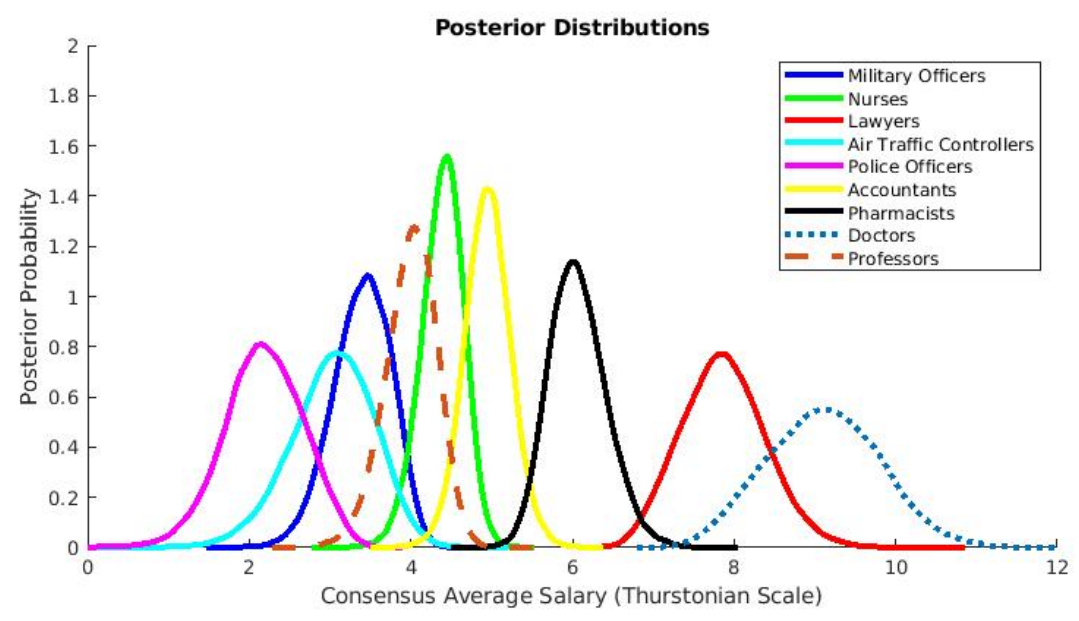

In this paper we discuss two separate models. The first model, referred to as the Strong Consensus Paired Comparison Model (SCPCM), reflects the essence of previous CCT models in that it assumes individuals always answer items from the same 
consensus "answer key", with each response carrying the possibility of independent error. We overview the model, strengths and shortcomings of this approach in the following sections. Later we will discuss an expansion of this model that relaxes these assumptions which we will refer to as the Weak Consensus model.

\section{Model 1: Strong Consensus}

The Strong CPCM (SCPCM) model is described according to the following axioms:

Axiom 1: (Common Truth). For group c of the $\mathrm{C}$ cultural groups, there is a row vector $T_{c}$ of latent values on the real line for each of the $\mathrm{M}$ items. $\mathrm{T}$ is a $C \times M$ matrix.

Axiom 2: (Thurstonian Paired Comparisons) The probability for subject i picking any item $\mathrm{A}$ over any item $\mathrm{B}$ is given by $P_{A \succ B}=\Phi\left(\frac{T_{g_{i} A}-T_{g_{i} B}}{\sqrt{\kappa_{i}\left(s_{g_{i}} A s_{g_{i} B}\right)}}\right)$. Where $\Phi$ is the inverse probit function, and $g_{i}$ indicates which cultural group c subject $\mathrm{i}$ is in. Note that this is equivalent to the Thurstonian case III model(Thurstone, 1927), where each Gaussian for item $\mathrm{k}$ is centered at $T_{g_{i} k}$ and has variance equal to $\kappa_{i} s_{g_{i} k}$.

Here, $\kappa_{i}$ can be seen as subject i's tendency to diverge from the group consensus, with larger values indicating more divergence whereas $s_{c k}$ can be seen as a measure of the level of inconsistency of people in group c's evaluation of item k, with larger values indicating less consistency.

In this strong consensus version of the model, it is assumed that everyone in the same group has the same latent value for each item, but individual subjects have 
different variances in their assessments of an items value.

Built into the assumptions of the case III Thurstonian model is weak stochastic transitivity on the individual level, and in the case of the SCPCM on the group level too, since all in group $\mathrm{c}$ share the same latent values $T_{c}$. What this means is that if $P_{A \succ B} \geq \frac{1}{2}$ and $P_{B \succ C} \geq \frac{1}{2}$, then $P_{A \succ C} \geq \frac{1}{2}$ for any of these probabilities from any individual in the same group c.

\subsection{Hierarchical Bayesian Parameter Estimation}

We use a hierarchical Bayesian approach to estimate the parameters in the model. Hierarchical Bayesian modeling involves specifying stronger distributional assumptions regarding not only the data itself, also the parameters involved in generating the data, yielding provably better estimates (Efron and Morris, 1977). In many cases one might opt to assume one single cultural group $(\mathrm{C}=1)$, but the following specification could be utilized in cases where more than one cultural group is assumed.

$$
\begin{aligned}
& g_{i} \sim \operatorname{Categorical}(\pi) \\
& \pi \sim \operatorname{Dirichlet}(L)
\end{aligned}
$$

Here $g_{i}$ can take any integer from 1 to C. $\pi$ in this case is a C dimensional vector of probabilities corresponding to the probability of a person being in each group, and is specified to come from a Dirichlet distribution parameterized by L. L can be set to equal a $\mathrm{C}$ dimensional vector of ones, which is essentially a $\mathrm{C}$ dimensional extension of the Uniform distribution. Alternatively, one can set L such that it gives the most weight to the first cultural group and assigns descending weight to the succeeding groups. 
The consensus value $\mathrm{T}$ for item $\mathrm{k}$ and cultural group $\mathrm{c}$ is given the following prior distribution:

$$
T_{c k} \sim N(5, .25)
$$

In this case, centering at 5 is completely arbitrary, chosen entirely for the sake of yielding values usually between 0 and 10 . Setting the precision (inverse variance) at .25 however is chosen so that the model can be properly identified.

Now both subject and item specific components of the Thurstonian variance are given lognormal priors, centered at 0 with uninformative gamma distributed hyperpriors for the precision:

$$
\begin{aligned}
& \log \left(\kappa_{i}\right) \sim N\left(0, \tau_{\kappa}\right) \\
& \log \left(s_{c k}\right) \sim N\left(0, \tau_{s}\right) \\
& \tau_{\kappa} \sim \operatorname{gamma}(.01, .01) \\
& \tau_{s} \sim \operatorname{gamma}(.01, .01)
\end{aligned}
$$

\subsubsection{Recovery Analysis for SCPCM}

Data was simulated from SCPCM for different numbers of subjects and items for a recovery analysis. Parameters used were drawn in accordance with the hierarchical specification, with both $\tau_{\kappa}$ and $\tau_{s}$ set to 1 . From these results we see that the model is successful in recovery, with better results when there are more subjects and items. Under fewer subjects and items, results are qualitatively accurate but are prone to some shrinkage from the hierarchical structure. 

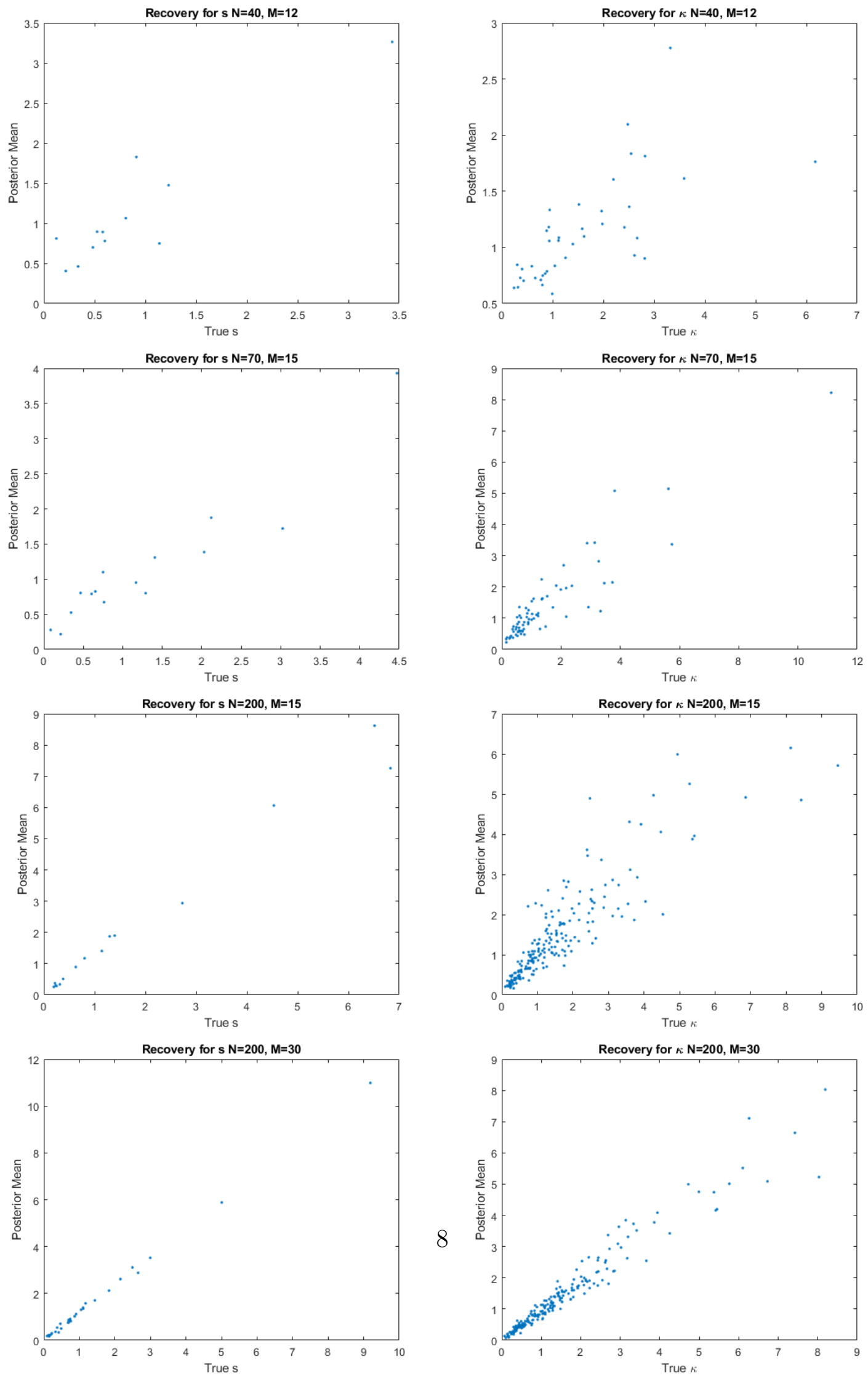


\section{Data Sets}

For each data set, an attempt was made to avoid repeating the same items too close to one another. In every case participants answered which item they choose between every possible pair of the set of items.

\subsection{Occupation Salaries}

40 subjects were asked to compare the salaries of 9 different occupations, such as pharmacist and police officer. Data was collected after a test in an undergraduate Psychology course, giving students the opportunity to fill out the survey in exchange for bonus points.

\subsection{Car Prices}

66 participants were shown pairs of pictures of 10 cars, with their make/model. Participants were asked which car they thought was more expensive of each pair. The study was conducted on computers, and participants were recruited through the schools online subject pool. Participants received credits through the subject pool that can usually be used for extra credit in some undergraduate Psychology courses.

\subsection{Cheerfulness of Paintings}

The same 66 participants that responded to Car Prices were also asked to compare which painting seemed more cheerful for each pair out of 15 different paintings. 

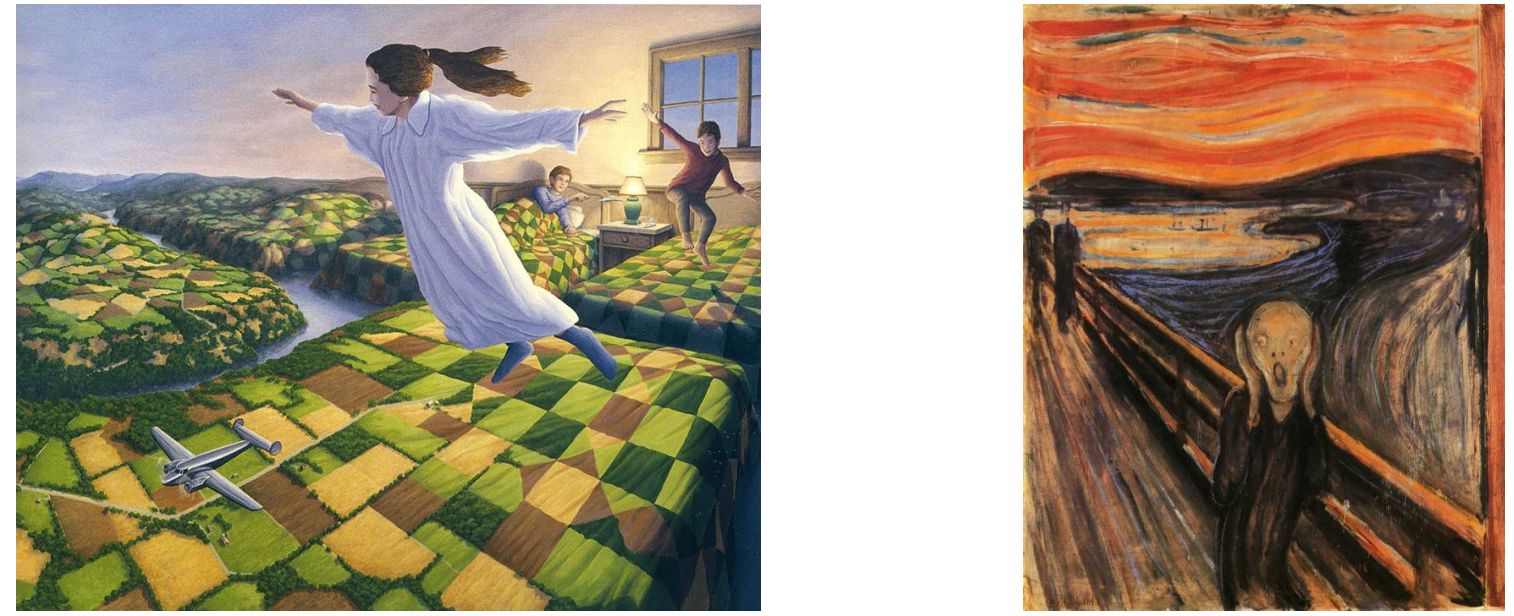

Figure 2: Two of the paintings used. Left: Bedtime Aviation by Rob Gonsalves, Right: The Scream by Edvard Munch

\section{Posterior Predictive Tests}

Since MCMC sampling samples from the joint posterior distribution of all parameters, we effectively get a set of possible parameter values for each iteration. We used each sample to construct a new simulated paired comparison data set. The simulated data sets allow for an extraction of a distribution for certain statistics of interest that we can compare with the observed statistics to check whether the model successfully captures the necessary information, using the same approach as in Gelman et al. (1996). Although these tests are traditionally referred to as posterior predictive tests, it should be noted that these distributions aren't actually predictions of new, untrained data, rather they represent the data you would expect to see if the model specification reflected the true generative stochastic process. Thus, it can be used as a tool to diagnose whether the model assumptions are inconsistent with observations, but not as tool to protect against overfitting. 


\subsection{Scree Smears}

Scree plots are constructed by doing a eigenvalue decomposition on the correlation matrix of the subject by item response matrix and plotting the values of the highest eigenvalues in a descending fashion. Previous CCT models have utilized Scree Plots primarily as a tool to detect the number of consensus answer keys (Anders et al., 2014), in this case corresponding to the value of $\mathrm{C}$ under axiom 1 . When $\mathrm{C}$ is one, one can expect a very high first eigenvalue followed by a sharp drop and quick plateau, whereas when $\mathrm{C}$ is two or more the sharp drop generally comes later. To test whether the model used is plausible, we compare the scree plots of the real data set with those of the simulated sets. We do this with a scree plot of the raw data, as well as using spearman rank-order correlation from the implied ranking through summation of all the times an item was preferred by each individual according to their responses.

\subsection{Violations of Transitivity}

If during the experiment a subject picked item A over B, B over C, and C over A, that subject has violated transitivity. From the simulated data sets, we can sum the total number of intransitivities over every triplet of items for every subject, and compare to the actual observed number. This measure can help inform us of whether the model reflects behavior on an individual level well.

\subsection{Between-Subject Transitivity Violations}

The previous check was effective in assessing the model's accuracy accounting for individual behaviors. But what about between people? This time, a violation of transitivity was counted if Subject A picked item i over $\mathrm{j}$, B picked item $\mathrm{j}$ over k, 
and $\mathrm{C}$ picked item k over i. All possible combinations and permutations of 3 people and 3 items were examined and summed over to calculate the total between person violations of transitivity. This is an interesting statistic to look at because if there is a high level of consensus on a set of items one would expect a low amount of between-subject transitivity violations, and vice versa.

\subsection{Posterior Predictive Results for the Strong Model}
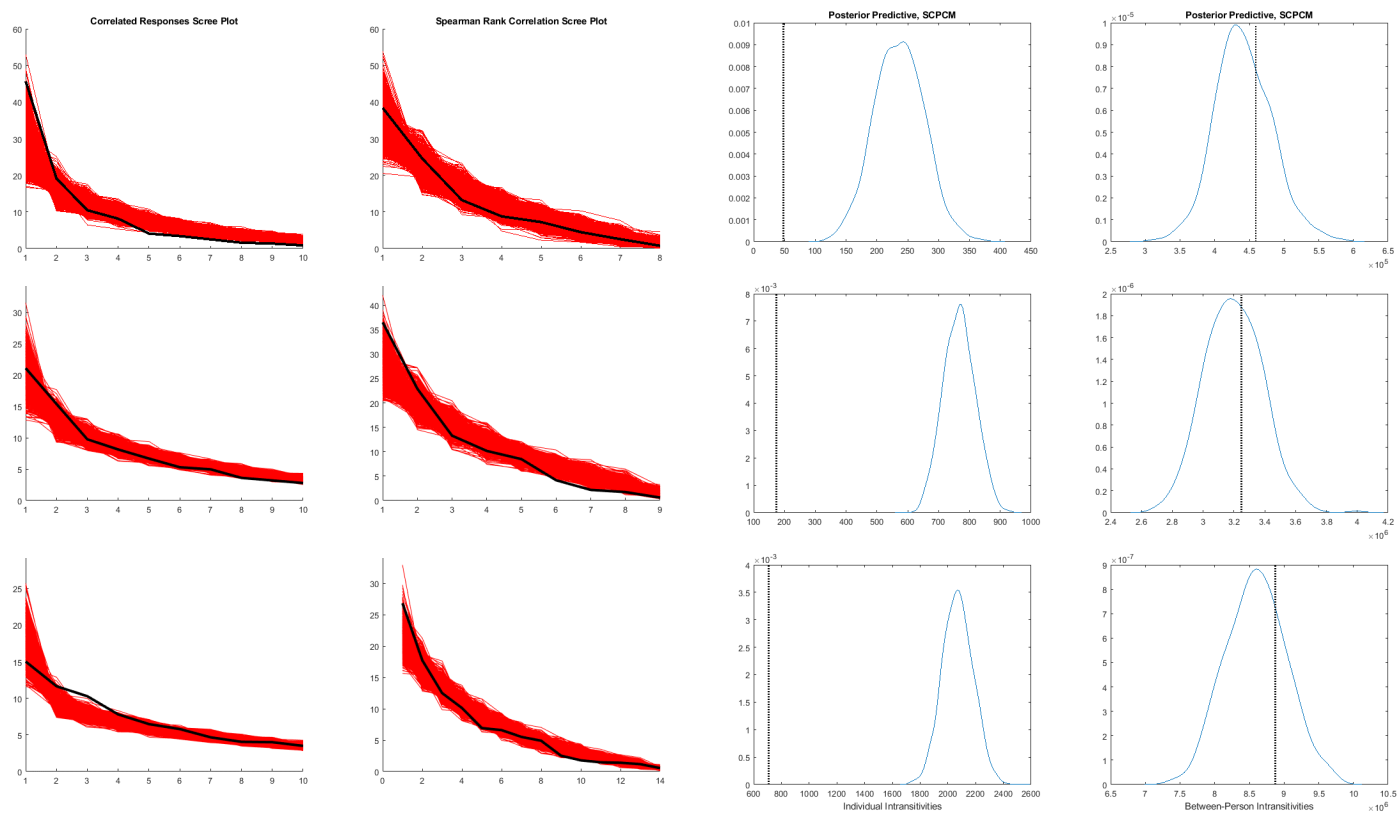

Figure 3: Posterior Predictive plots for the SCPCM fit to the occupations (first row), cars (second row), and paintings (third row) datasets.

In these simulations we see that the model does a decent job with most of the posterior predictive statistics, but it consistently drastically overestimates the number of individual transitivity violations that should occur. Apart from that, only the Pearson scree plot for the painting cheerfulness task seems a bit off. While this model 
seems to be mostly consistent with response phenomena at a larger group level, it could use some improvement for accounting for individual response behavior.

The reasoning behind this discrepancy was considered. The Strong Consensus Model assumes that everybody has the same underlying ground truth, and each time people evaluate the value of an item they are drawing from a distribution centered in the same place as it is for everyone else. This assumption implies that every time someone makes a decision that greatly diverges from consensus, they would have been just as likely to diverge from consensus in the opposite direction. In other words, the model treats divergence from consensus to be the same as a lack of self-consistency, this seems like the likely source of the model's inconsistency with observations. To account for this inconsistency we developed the Weak Consensus Paired Comparison Model (WCPCM).

\section{Axioms of the Weak Consensus Model}

The weak consensus model differs from the strong consensus model in that it assumes that people do not necessarily share precisely the same latent opinion on the values of the items, but people in the same group will have latent opinions on the values of the items that are similar to one another. In essence, one can think

of the WCPCM as the SCPCM with an extra layer between group consensus and deliberation, accounting for underlying disagreement.

Axiom 1: (Common Truth). For group c of the $\mathrm{C}$ cultural groups, there is a row vector $T_{c}$ of latent values on the real line for each of the $\mathrm{M}$ items. $\mathrm{T}$ is a $C \times M$ matrix. 
Axiom 2: (Individual Latent Item Values). Subject i has a fixed latent item value for item $\mathrm{k}$ given by $Y_{i k}=T_{g_{i} k}+\epsilon_{i k}$, where $\epsilon_{i k} \sim N\left(0, \frac{1}{E_{i} \lambda_{g_{i}}}\right)$ (precision notation) and $g_{i}$ indicates which cultural group c subject $\mathrm{i}$ is in.

Axiom 3: (Thurstonian Paired Comparisons) The probability for subject i picking any item $\mathrm{A}$ over any item $\mathrm{B}$ is given by $P_{A \succ B}=\Phi\left(\frac{Y_{i A}-Y_{i B}}{\sqrt{\kappa_{i}\left(s_{g_{i} A}+s_{g_{i} B}\right)}}\right)$. Where $\Phi$ is the inverse probit function . Note that this is equivalent to the Thurstonian case III model(Thurstone, 1927), where each Gaussian for item $\mathrm{k}$ is centered at $Y_{i k}$ and has variance equal to $\kappa_{i} s_{g_{i} k}$.

It should be noted that $E_{i}$ can be seen as a measure of subject i's tendency to view items as having a value close to his/her cultural group $g_{i}$, with small $E_{i}$ s denoting a tendency of being closer to their cultural group. Likewise, $\lambda_{c k}$ can be seen as the variability in people's latent item values across different people in cultural group $c$ for item $\mathrm{k}$.

Similarly, $\kappa_{i}$ can be viewed as a measure of individual i's consistency in their assessment of item value for the same item, with lower values indicating more consistency. $s_{g_{i} k}$ Can be viewed as the tendency of item $\mathrm{k}$ in being evaluated consistently for cultural group $g_{i}$, with lower values indicating more consistency in evaluation for that item within an individual's evaluations.

This time, weak stochastic transitivity is again followed as a consequence of the case III Thurstonian model, but only on the individual level, since individuals do 
have different latent values for the items.

\subsection{Hierarchical Bayesian Parameter Estimation}

The Hierarchical specification of the WCPCM is identical to the SCPCM, only with the addition of the $\lambda$ and $E$ terms which are given the same sort of hyperprior as the $\kappa$ and $s$ terms:

$$
\begin{aligned}
& \log \left(\lambda_{c k}\right) \sim N\left(0, \tau_{\lambda}\right) \\
& \log \left(E_{i}\right) \sim N\left(0, \tau_{E}\right) \\
& \tau_{\lambda} \sim \operatorname{gamma}(.01, .01) \\
& \tau_{E} \sim \operatorname{gamma}(.01, .01)
\end{aligned}
$$

\subsubsection{Recovery Analysis for Weak Model}

Similar to before, data was simulated according to the model with all the various $\tau \mathrm{s}$ set to 1 . We can see here that despite not being as accurate in terms of estimating the exact raw parameters (being a more complex model), the qualitative results are approximately right and get better with more participants and more items. 

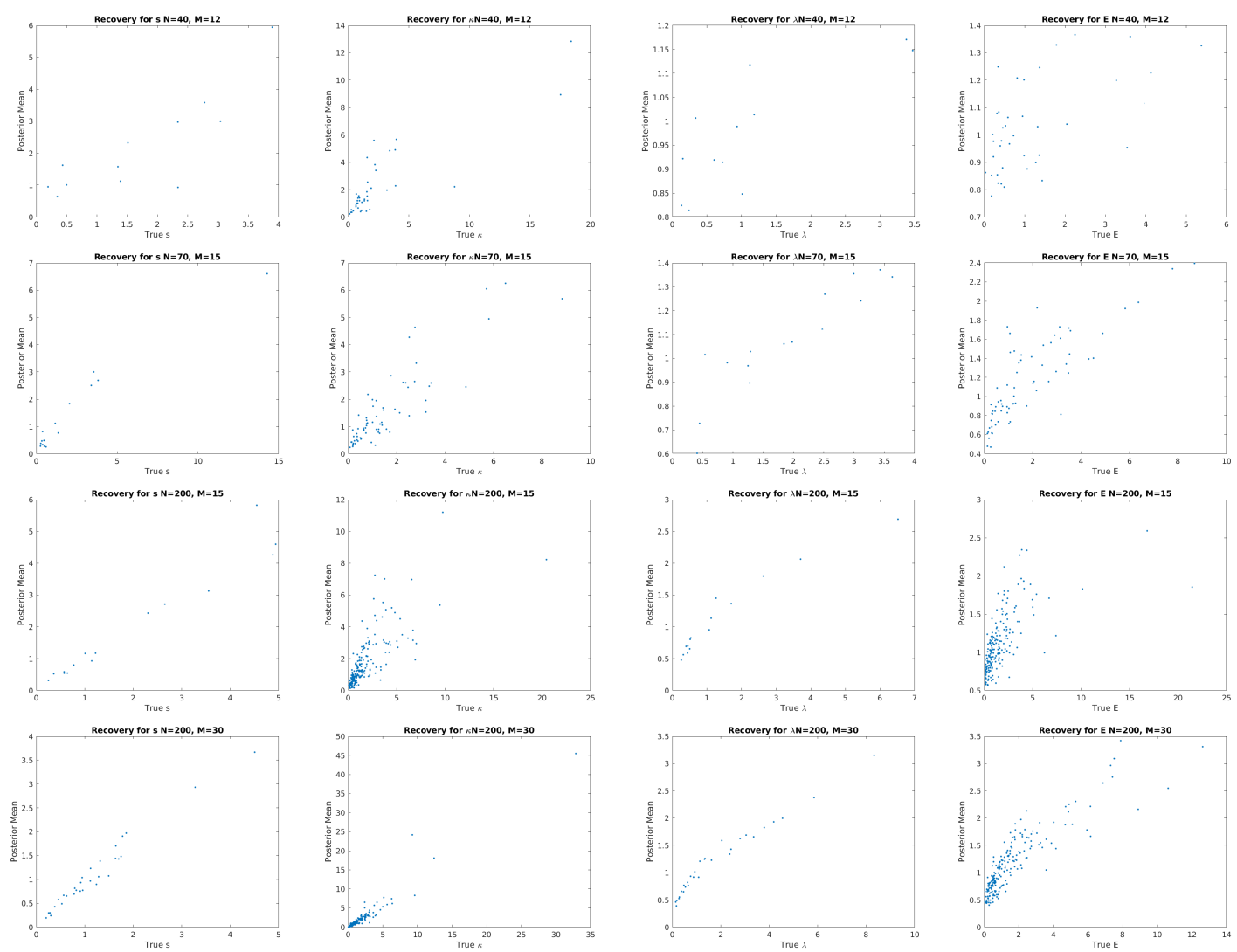

\subsubsection{Do Items Really Vary in Individual Consistency?}

It's not immediately apparent that $s_{g_{i} k}$ is really a necessary parameter. One could set them all equal to 1 and then that would only leave a measure for individual self-consistency and it might work just as well. A Bayes Factor was used to test this for the three data sets, giving 50 percent prior probability to all of the $s_{g_{i} k} \mathrm{~s}$ equaling 1, and 50 percent prior probability for the full weak consensus model. The Bayes Factors came out just barely in favor of the full weak consensus model for the paintings and occupations, which came out to be 1.01 and 1.56 respectively. For the car prices set however, evidence for the full weak consensus model was very strong, 
with a Bayes Factor of 93.3. You can see the results summarized in the table below.

\begin{tabular}{|c|c|c|c|c|c|c|}
\hline Model & Price & $\begin{array}{l}\text { WC Mean } \\
\text { Value }\end{array}$ & SC Mean Value & $\begin{array}{l}\text { WC } \\
\text { Disagreement }\end{array}$ & $\begin{array}{l}\text { WC } \\
\text { Inconsistency }\end{array}$ & SC "Difficulty" \\
\hline Toyota Camry LE & 22520 & 3.0217 & 4.1075 & 0.7482 & 0.284 & 0.5485 \\
\hline Porsche 718 Boxter & 58450 & 9.2753 & 6.767 & 0.9567 & 1.8489 & 0.6046 \\
\hline Acura ILX & 29065 & 3.8795 & 4.5772 & 1.0312 & 0.143 & 0.5085 \\
\hline Subaru Outback & 25053 & 3.7294 & 4.5229 & 1.3327 & 0.4239 & 0.4962 \\
\hline Jeep Wrangler & 25090 & 5.2976 & 5.1906 & 1.3729 & 0.4258 & 0.5003 \\
\hline Audi R8 & 166150 & 8.7639 & 6.435 & 1.1779 & 2.4979 & 0.5768 \\
\hline $\begin{array}{l}\text { Ford F150 Regular Cab 2WD } \\
6.5^{\prime}\end{array}$ & 26977 & 3.8854 & 4.5368 & 1.5147 & 0.4178 & 0.5339 \\
\hline Mazda MAZDA3 & 17862 & 3.7915 & 4.5675 & 1.072 & 0.2136 & 0.4992 \\
\hline Honda Civic & 19267 & 3.0383 & 4.1539 & 0.7376 & 0.6615 & 0.5645 \\
\hline Land Rover Discovery & 53085 & 6.1368 & 5.5125 & 1.2351 & 0.5491 & 0.5047 \\
\hline
\end{tabular}

As we can see there were certain cars that people were substantially more inconsistent with than others. Interestingly this was strongest for the two most expensive cars, both of which happened to be sports cars. What's more, we see with the Strong consensus model almost no difference in difficulty in assessment, which might be interpreted to indicate that there is about the same level of agreement about each car, but the results from the Weak Consensus model tell a different story about the varying levels of agreement. From the Weak consensus model we can see that there was more disagreement surrounding the prices of the pickups and SUVs, while the more generic cars like the Toyota Camry and Honda Civic had more agreement surrounding their prices. This would go unnoticed if we stuck to the strong consensus model. 


\subsection{Posterior Predictive Tests for the Weak Consensus Model}
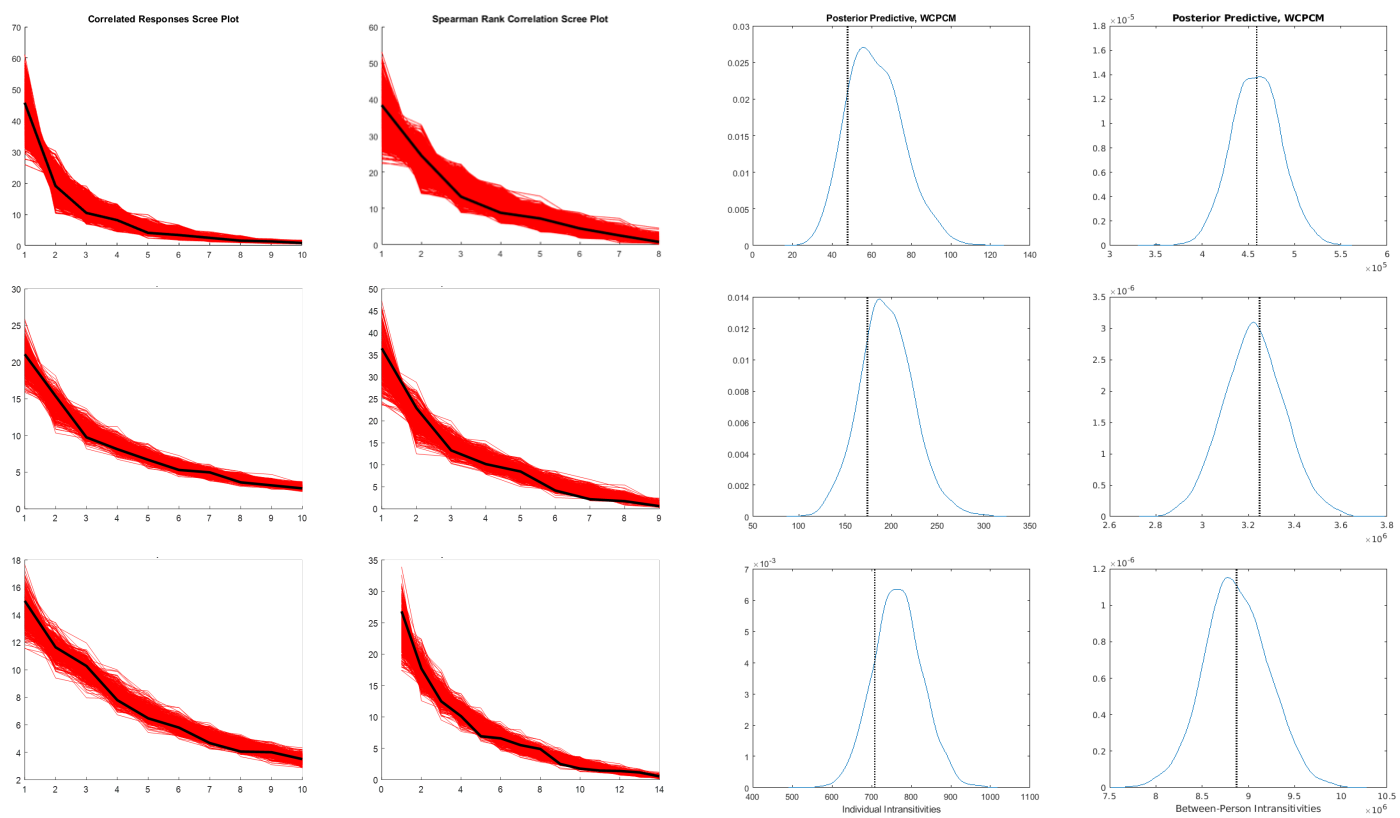

Figure 5: Posterior Predictive plots for the WCPCM fit to the occupations (first row), cars (second row), and paintings (third row) datasets.

It can be seen that every issue with the posterior predictive checks that was present in the strong consensus model is not present in the weak consensus model. We see true statistics represented in reasonable locations in the posterior predictive distributions.

\section{Discussion}

The SCPCM and WCPCM presented in this paper are able to serve as effective tools in the analysis of paired comparison data, quantifying the consensus values of a set of items on a Thurstonian scale. While the SCPCM makes stronger assumptions that might be less realistic (as illustrated in posterior predictive checks), its 
simplicity and better identifiability still make it worthy of use for some applications. The WCPCM's relaxation of assumptions and inclusion of additional measures can make it a more attractive choice when there is a sufficient number of items and participants. Indeed, as we saw that in the car prices dataset, the WCPCM picked up on properties regarding levels of agreement on prices between different kinds of automobiles that were non-emergent from the SCPCM analysis, along with tendencies regarding consistency in item analysis. By treating people's variability in assessment in their own choices as separate from their tendency to respond in accordance with group consensus, we are able to uncover the full story more.

A limitation of these models is that they fail to account for any potential pairwise context effects since they utilize the case III Thurstonian model. This is a potential avenue for an extension of the currently proposed approach. For example, one might include additive terms to either the $\mathrm{T}$ or the $\mathrm{Y}$ terms that are only added in the presence of specific alternatives to test for context effects under this framework. The power and robustness of such approaches to testing context effects under this framework are worthy of exploration. It may also be feasible to utilize the Case I Thurstonian model for these purposes, which models latent appraisals from paired comparisons as coming from multivariate normal distributions as opposed to two independent gaussians.

As with other CCT models, it would be worthwhile to dedicate more formal exploration into their potential for wisdom of the crowds applications. The SCPCM as introduced here shares many similarities with Lee et al. (2012), which was designed for these purposes. While Lee et al. (2012) used rank-order data and the SCPCM and WCPCM use paired-comparison data, it might be interesting to compare these mod- 
els in their usefulness for wisdom of the crowds applications. Preliminary work into using a CCT approach for wisdom of the crowds applications such as in Alexander (2017) has shown promising results, thus it is worthy of further exploration.

\section{Acknowledgments}

Thanks to Jeffrey Rouder for discussion and input. This project was funded by NSF grant \# 1534471.

\section{References}

Alexander, G.E., 2017. A Mathematical Explication of Human Psychology. Ph.D. thesis.

Anders, R., Batchelder, W.H., 2015. Cultural consensus theory for the ordinal data case. Psychometrika 80, 151-181.

Anders, R., Oravecz, Z., Batchelder, W., 2014. Cultural consensus theory for continuous responses: A latent appraisal model for information pooling. Journal of Mathematical Psychology 61, 1-13.

Batchelder, W.H., Anders, R., 2012. Cultural consensus theory: Comparing different concepts of cultural truth. Journal of Mathematical Psychology 56, 316-332.

Batchelder, W.H., Anders, R., Oravecz, Z., 2018. Cultural consensus theory. Stevens' Handbook of Experimental Psychology and Cognitive Neuroscience 5, 201-264. 
Batchelder, W.H., Strashny, A., Romney, A.K., 2010. Cultural consensus theory: Aggregating continuous responses in a finite interval, in: International Conference on Social Computing, Behavioral Modeling, and Prediction, Springer. pp. 98-107.

Efron, B., Morris, C., 1977. Stein's paradox in statistics. Scientific American 236, 119-127.

Gelman, A., Meng, X.L., Stern, H., 1996. Posterior predictive assessment of model fitness via realized discrepancies. Statistica sinica , 733-760.

Lee, M.D., Steyvers, M., De Young, M., Miller, B., 2012. Inferring expertise in knowledge and prediction ranking tasks. Topics in cognitive science 4, 151-163.

Romney, A.K., Weller, S.C., Batchelder, W.H., 1986. Culture as consensus: A theory of culture and informant accuracy. American anthropologist 88, 313-338.

Thurstone, L.L., 1927. A law of comparative judgment. Psychological review 34, 273. 